\title{
Hidrógeno mediante reformado catalítico de biogás en reactor de lecho fluidizado de dos zonas con membranas permeoselectivas
}

\author{
Patricia Ugarte, Paúl Durán, Javier Lasobras, Javier Herguido, Miguel Menéndez \\ Grupo de Catálisis, Separaciones Moleculares e Ingeniería de Reactores (CREG) \\ Instituto de Investigación en Ingeniería de Aragón (I3A) \\ Universidad de Zaragoza, Mariano Esquillor s/n, 50018, Zaragoza, Spain. \\ Tel. +34-976762707, e-mail:pugarte@unizar.es
}

\begin{abstract}
En esta investigación se plantea el uso de biogás para la obtención de hidrógeno en un reactor de lecho fluidizado de dos zonas. Se ha comprobado que el uso simultáneo de membranas de $\mathrm{Pd} / \mathrm{Ag}$, permeoselectivas para retirar hidrógeno, permite obtener rendimientos mucho mayores de los reportados en bibliografía.
\end{abstract}

\section{Introducción}

Este proyecto aborda el uso de una fuente energética no fósil y de carácter renovable como es el biogás para la producción de hidrógeno, utilizable como vector energético[1]. Se enmarca en el reto global del abastecimiento sostenible y respetuoso con el medio ambiente, además de contribuir a la eliminación controlada de residuos mayormente de carácter antropogénico [2]. En este trabajo, se combinan procesos de reacción catalítica + separación + regeneración del catalizador en un único reactor; gracias a la utilización de reactores de lecho fluidizado de dos zonas [3] y de membranas permeoselectivas al $\mathrm{H}_{2}$ (RLFDZ+MB).[4]

\section{Experimental}

Se han sintetizado cuatro catalizadores soportados en $\mathrm{Al}_{2} \mathrm{O}_{3}$, cambiando la carga activa del metal: $\mathrm{Ni}$ ( 5 $\% w t.), \mathrm{Ni}(10 \% w t),. \mathrm{Ni}+\mathrm{Ce}(5+10 \% \mathrm{wt}),. \mathrm{Ni}+\mathrm{Co}$ $(5+10 \% w t$.$) . Todos ellos se han preparado por el$ método de impregnación a humedad incipiente y se han caracterizados mediante XRD, XRF y área BET. Los experimentos realizados se han clasificado en función del tipo de reactor utilizado. Primero, un estudio de catalizadores en una configuración de lecho fluidizado convencional (15 $\mathrm{g}$ catalizador, $\left.u_{o}=2 u_{m f}\right)$. En una segunda fase se introdujeron en el reactor membranas de $\mathrm{Pd} / \mathrm{Ag}$ para separar hidrógeno. Para finalizar, se hicieron experimentos con el RLFDZ y con membranas (30 $\mathrm{g}$ catalizador, $u_{o}=3 u_{m f}$ parte superior; $u_{o}=1,2 u_{m f}$ parte inferior). La presión parcial del biogás se varía pero manteniendo siempre la proporción $\mathrm{CH}_{4}: \mathrm{CO}_{2}$ en $1: 1$.

\section{Resultados}

Se hizo primeramente un estudio de actividad de los catalizadores en reactor de lecho fluidizado convencional, en el que se obtuvieron resultados muy similares para todos ellos. Los experimentos realizados en configuración RLFDZ+MB son los más representativos de este trabajo. En la Figura 1 se puede ver un experimento realizado con catalizador $\mathrm{Ni}+\mathrm{Ce}(5+10 \% \mathrm{wt}$ ), estando dividido en 4 etapas. En la Etapa 1 se opera en RLF, en la Etapa 2 se cambia a reactor de dos zonas (RLFDZ) y se usa $\mathrm{CO}_{2}$ como regenerante. En la Etapa 3 se extrae hidrógeno por las membranas (RLFDZ+MB) y en la última manteniendo la configuración anterior $(\mathrm{RLFDZ}+\mathrm{MB})$ se cambia el regenerante a $\mathrm{O}_{2}$. A partir de la Etapa 2 se aprecia un aumento de conversión al extraer hidrógeno por el desplazamiento del equilibrio. También se observa que, independientemente del regenerante la conversión es la misma (Etapas 3 y 4). Esto llevó a realizar experimentos con ambos regenerantes en distintas proporciones (Figura 2). Se observa que con el menor porcentaje de $\mathrm{O}_{2}$ usado, no se regenera lo suficiente ya que, al aumentar su proporción, aumenta la conversión. El uso de $\mathrm{CO}_{2}$ como regenerante, en una proporción que equipare el poder oxidante del $\mathrm{O}_{2}$ (el doble del $\mathrm{CO}_{2}$ ) muestra que el sistema se comporta igual que para el caso de un $3,4 \%$ de oxígeno. Por lo tanto, el $\mathrm{CO}_{2}$ puede utilizarse como agente regenerante para este proceso. $\mathrm{La}$ intensificación alcanzada permite aumentar la conversión de $\mathrm{CH}_{4}$ y $\mathrm{CO}_{2}$ y los ratios $\mathrm{H}_{2} / \mathrm{CO}$ en productos mejoran ampliamente los de bibliografía como se puede ver en la Figura 3.

\section{Conclusiones}

La reacción de reformado seco de biogás en reactores de lecho fluidizado convencional se puede llevar a cabo con valores de conversión cercanos al equilibrio y sin observar desactivación del catalizador en el periodo de reacción. Todos los catalizadores sintetizados muestran el mismo comportamiento (su diferenciación viene dada por 
el producto que se considere de interés) y todos mantienen sus propiedades y estructuras tras varios ciclos de reacción. Se encontró que el sistema en su configuración con membranas necesita un periodo de activación en el cual no se extrae hidrógeno, para no tener pérdida de actividad en el catalizador por encontrarse en una atmósfera muy oxidante al principio de reacción. En cuanto al sistema RLFDZ+MB, se puede destacar que se obtuvieron conversiones más altas que las obtenidas en configuraciones análogas sin membranas y que el sistema funcionó de manera estable durante todo el tiempo de operación. Se ha podido demostrar que el $\mathrm{CO}_{2}$ es una alternativa como agente regenerante respecto al oxígeno, obteniendo tendencias y valores similares de conversión ratio y selectividad.

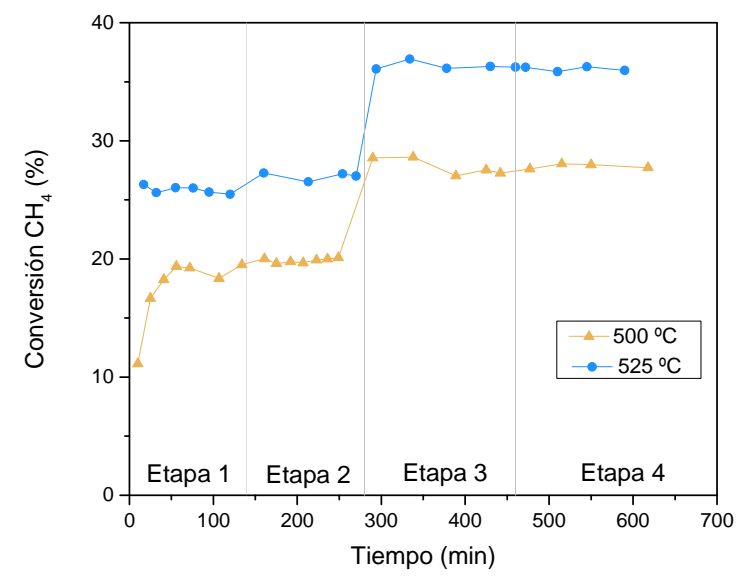

Figura 1. Evolución de la conversión de metano para los experimentos en 4 etapas de RSM en RLFDZ+ $\mathrm{MB}, \mathrm{a} 500$ y $525{ }^{\circ} \mathrm{C}$.

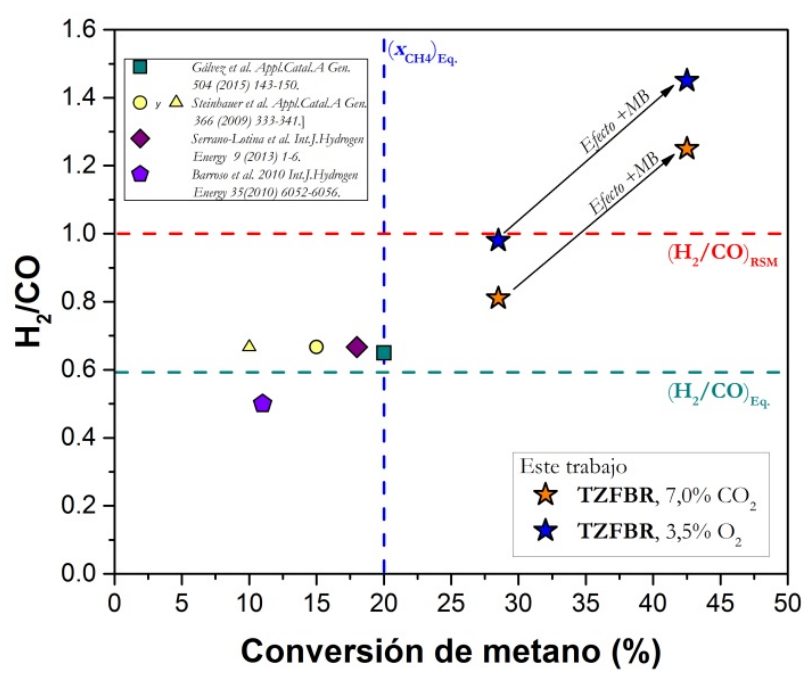

Figura 3. Comparación de los resultados obtenidos en este trabajo con los reportados en literatura para catalizadores de níquel y ratio $\mathrm{CH}_{4}: \mathrm{CO}_{2}$ 1:1

\section{REFERENCIAS}

[1] PLOU, J., DURÁN, P., HERGUIDO, J., PEÑA, J.A. Purified hydrogen from synthetic biogas by joint methane dry reforming and steam-Iron process: Behaviour of metallic oxides and coke formation. Fuel 2014, 118, 100.

[2] USMAN, M., WAN DAUD, W.M., ABBAS, H.F.Dry reforming of methane: Influence of process parameters-A review. Renew. Sustain Energy Rev 2015, 45, 710.

[3] HERGUIDO, J., MENÉNDEZ, M., SANTAMARÍA, J. On the use of fluidized bed catalytic reactors where reduction and oxidation zones are present simultaneously. Catal. Today 2005, $100,181$.

[4] MEDRANO, J.A., JULIÁN, I., GARCÍA-GARCÍA, F.R., LI, K., HERGUIDO, J., MENÉNDEZ, M. Two zone fluidized bed reactor (TZFBR) with palladium membrane for catalytic propane dehygrogenation: Experimental performance assessment. Ind. Eng. Chem. Res 2013, 52, 3723.

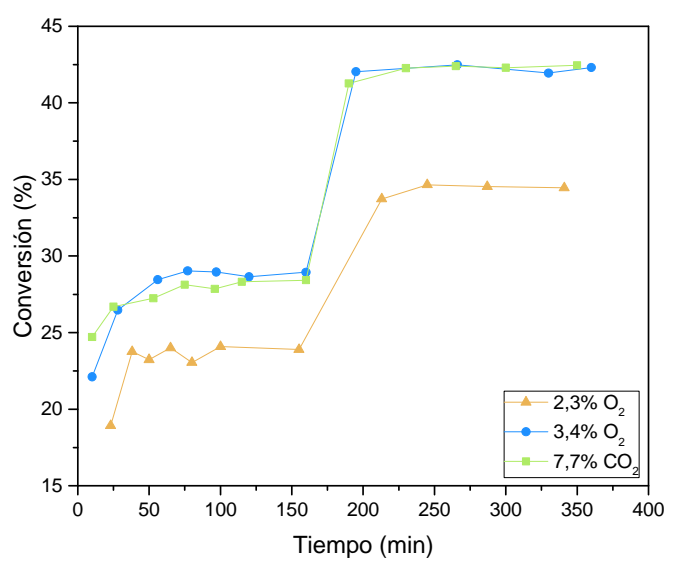

Figura 2. Evolución de la conversión para los experimentos con cambios en naturaleza $y$ proporción del agente regenerante a $500{ }^{\circ} \mathrm{C}$. 